bioRxiv preprint doi: https://doi org/10.1101/2021.11.24.469880; this version posted November 29,2021 . The copyright holder for this preprint

(which was not certified by peer review) is the author/funder, who has granted bioRxiv a license to display the preprint in perpetuity. It is made available under aCC-BY-NC-ND 4.0 International license.

\title{
Asymmetric and adaptive reward coding via normalized reinforcement learning
}

Kenway Louie

Center for Neural Science, New York University, New York, United States

Neuroscience Institute, New York University Grossman School of Medicine, New York, United States

Corresponding author:

Kenway Louie

Center for Neural Science

New York University

4 Washington Place, Room 6

New York, NY 10003

Voice: +1.212 .998 .3904$

Fax: +1.212.995.4011

Email:klouie@cns.nyu.edu 


\begin{abstract}
1 Abstract
2

3 Learning is widely modeled in psychology, neuroscience, and computer science by prediction error4 guided reinforcement learning (RL) algorithms. While standard RL assumes linear reward functions, 5 reward-related neural activity is a saturating, nonlinear function of reward; however, the computational 6 and behavioral implications of nonlinear RL are unknown. Here, we show that nonlinear RL 7 incorporating the canonical divisive normalization computation introduces an intrinsic and tunable 8 asymmetry in prediction error coding. At the behavioral level, this asymmetry explains empirical 9 variability in risk preferences typically attributed to asymmetric learning rates. At the neural level, 10 diversity in asymmetries provides a computational mechanism for recently proposed theories of 11 distributional RL, allowing the brain to learn the full probability distribution of future rewards. This 12 behavioral and computational flexibility argues for an incorporation of biologically valid value functions 13 in computational models of learning and decision-making.
\end{abstract}




\section{Intoduction}

Reinforcement learning (RL) provides a theoretical framework for how an agent learns about its environment and adopts actions to maximize its cumulative long-term reward (Sutton \& Barto, 1998). In standard RL models, the values of stimuli or actions are learned via a reward prediction error (RPE), defined as the difference between actual and expected outcomes. Using RPE signals over repeated samples from the environment, learners progressively update their estimates to obtain a measure of the average value (e.g. of an action, a state, or a state-action pair). For example, in its simplest form an RL model updates its value estimate $V_{t+l}$ as:

$$
V_{t+1}=V_{t}+\eta\left(R_{t}-V_{t}\right)
$$

where the incremental update signal comprises a learning rate $\eta$ and an RPE term. The canonical RPE term is the difference between actual reward $R_{t}$ and expected reward $V_{t}$, which produces value signals that are a linear function of reward. RL algorithms accurately capture various types of learning in animal and human subjects (Botvinick, Niv, \& Barto, 2009; Dolan \& Dayan, 2013), and have been employed in increasingly complex ways to produce powerful artificial intelligence agents (Mnih et al., 2015; Silver et al., 2017; Song, Yang, \& Wang, 2017). Furthermore, the activity of midbrain dopamine neurons closely matches theoretical RPE signals (Bayer \& Glimcher, 2005; Cohen, Haesler, Vong, Lowell, \& Uchida, 2012; Schultz, Dayan, \& Montague, 1997), suggesting that RL serves as a computational model of neurobiological learning.

Despite their diversity and ubiquity, almost all RL approaches utilize a linear reward function at odds with evidence for nonlinear reward coding in the brain. A nonlinear relationship between internal subjective value and external objective reward is a longstanding assumption in psychology and economics. For example, risk preferences in choice under uncertainty are consistent with choosers employing a utility function that is a nonlinear function of reward (Luce, 1959; Von Neumann \& Morgenstern, 1944). This behavioral nonlinearity is reflected in underlying neural responses, with activity in reward-related brain areas correlating with subjective values rather than objective reward amounts (Levy, Snell, Nelson, Rustichini, \& Glimcher, 2010; Padoa-Schioppa \& Assad, 2006; Stauffer, Lak, \& Schultz, 2014). Notably, in contrast to standard RL assumptions, the activity of dopamine neurons also exhibits a nonlinear response consistent with a subtraction between actual and expected reward terms, both of which are sublinear functions of reward amount (Eshel et al., 2015; Eshel, Tian, Bukwich, \& Uchida, 2016). Together, these results suggest that neurobiological RL mechanisms operate on nonlinear reward representations, but the behavioral and computational implications of nonlinear RL are not well understood.

Here, we develop and characterize an RL algorithm that learns a nonlinear reward function implemented via the divisive normalization computation (Heeger, 1992). Originally proposed to describe nonlinear responses in early visual cortex, normalization has been observed in multiple brain regions, sensory modalities, and species, suggesting that normalization is a canonical neural computation (Carandini \& Heeger, 2012). In addition to sensory processing, normalization explains neural responses in higher-order cognitive processes including attention, multisensory integration, and decision-making (Louie, Grattan, \& Glimcher, 2011; Ohshiro, Angelaki, \& DeAngelis, 2017; Reynolds \& Heeger, 2009; Sheahan, Luyckx, Nelli, Teupe, \& Summerfield, 2021). In particular, its role in reward coding makes it an attractive candidate mechanism for nonlinear reward representations in reinforcement learning.

\section{Results}

Normalized reinforcement learning model 
In contrast to standard RL, we propose a normalized RL algorithm (NRL) that learns a value function nonlinearly related to objective reward. Specifically, NRL (Fig. 1A) assumes that objective rewards $R$ are represented by a normalized subjective value function $U$ :

$$
U(R)=\frac{R^{n}}{\sigma^{n}+R^{n}}
$$

and that learning employs the corresponding normalized prediction error term:

$$
V_{t+1}=V_{t}+\eta\left(U\left(R_{t}\right)-V_{t}\right)=V_{t}+\eta\left(\frac{R_{t}^{n}}{\sigma^{n}+R_{t}^{n}}-V_{t}\right)
$$

where the exponent and semisaturation parameters $n$ and $\sigma$ govern the precise form of the nonlinear transformation (see below). Normalization produces a value function that is a saturating function of reward: at small reward magnitudes $(R<<\sigma)$ value grows with reward, while at large reward magnitudes $(R>>\sigma)$ value approaches an asymptote. Consistent with this intuition, in simulation NRL algorithms learn values that are a saturating nonlinear function of reward (Fig. 1B).

Unlike standard nonlinear utility functions, normalized value functions can exhibit an inflection point in reward responses that introduces an intrinsic, magnitude-dependent asymmetry in RPEs. The direction and extent of this asymmetry depends on the specific parameterization of the normalized value function. For $n \leq 1$, normalized value functions are always concave; however, for $n>1$, normalized value functions are convex at lower rewards and concave at higher rewards (Fig 1C); convex (concave) regimes are evident as positive (negative) regions of the normalized value second derivative. In the convex regime, increases in $R$ generate larger changes in $U(R)$ than equivalent decreases in $R$; this predicts asymmetric RPE responses biased towards outcomes better than expected. In contrast, in the concave regime, decreases in $R$ generate larger $U(R)$ changes and RPEs are biased towards outcomes worse than expected. Notably, both theoretical and empirical considerations support normalization exponents consistent with inflection points: input squaring $(n=2)$ was used to model threshold linear responses in spiking activity in the original normalization equation(Heeger, 1992), and fits of $n$ to neural data typically yield values between 1.0 and 3.5 (average value of 2) (Busse, Wade, \& Carandini, 2009; Carandini \& Heeger, 2012). Thus, asymmetric RPE responses are likely to arise if reward learning relies on normalized value coding.

Critically, the type of NRL RPE asymmetry is parametrically tunable and depends on the relationship between rewards and the semisaturation term $\sigma$. Value function convexity and resulting positive-biased RPE asymmetry arise for rewards less than $\sigma$, while value concavity and negative-biased RPE asymmetry arise for rewards greater than $\sigma$. When $\sigma$ is varied, the reward amounts generating value concavity and convexity shift accordingly (Fig. 1D; see Appendix). As a result, asymmetric predictions errors around a given reward magnitude can be either negatively or positively biased, depending on the magnitude of the semisaturation term (Fig. 2). Thus, the degree and direction of RPE asymmetry is parametrically controlled in the NRL algorithm; we next examine how variability in this asymmetry can generate variability in risk preferences (across individuals) and in reward learning (across information processing channels).

\section{Variability in risk preference}

RPE asymmetry predicts that reinforcement learners will differentially weight outcomes that are better or worse than expected, consistent with behavior in multiple empirical studies (Frank, Moustafa, Haughey, Curran, \& Hutchison, 2007; Frank, Seeberger, \& O'Reilly R, 2004; Lefebvre, Lebreton, Meyniel, Bourgeois-Gironde, \& Palminteri, 2017; Niv, Edlund, Dayan, \& O'Doherty, 2012). However, previous studies - assuming linear reward coding - attributed this asymmetry to different learning rates for positive 
versus negative RPEs. For example, variable risk preferences in choice under uncertainty can be captured by standard RL models with valence-dependent learning rates (Mihatsch \& Neuneier, 2002):

$$
V_{t+1}=\left\{\begin{array}{l}
V_{t}+\eta^{+}\left(R_{t}-V_{t}\right) \text { if }\left(R_{t}-V_{t}\right)>0 \\
V_{t}+\eta^{-}\left(R_{t}-V_{t}\right) \text { if }\left(R_{t}-V_{t}\right)<0
\end{array}\right.
$$

For choices between a certain option and an uncertain option with the same mean outcome, these different learning rates implement a risk-sensitive RL process. If $\eta^{-}>\eta^{+}$, the model will learn an uncertain option value lower than its mean nominal outcome and exhibit risk-aversion; in contrast, if $\eta^{-}<\eta^{+}$, the model will overestimate uncertain options and exhibit risk-seeking behavior. RL models with differential learning rates have been increasingly influential and examined in the context of their adaptive properties (Caze \& van der Meer, 2013; Gershman, 2015), role in cognitive biases (Lefebvre et al., 2017; Palminteri, Lefebvre, Kilford, \& Blakemore, 2017), and potential neurobiological substrates (Daw, Kakade, \& Dayan, 2002; Frank et al., 2007; Frank et al., 2004).

Here, we show that the NRL model can generate variable risk preferences without requiring different learning rates. We simulated NRL behavior in a dynamic learning and choice task, in which the value of certain and risky options had to be learned via outcomes (Niv et al., 2012). Given the relationship between $\sigma$ and RPE asymmetry, the NRL model can generate either risk averse or risk seeking behavior (Fig. 3A, blue lines). Across a population of simulated subjects $(N=50), \mathrm{NRL}$ agents generated a range of behavioral risk aversion levels that are strongly correlated with $\sigma\left(r=-0.889 ; p=7.08 \times 10^{-18}\right)$. However, if a linear reward function is wrongly assumed, RL models with differential learning rates (Eqn. 4) can accurately fit NRL-generated behavior (Fig. 3A, black lines); furthermore, these data will appear to support differential learning rates linked to risk preference (Fig. 3A, right). Intuitively, these apparent learning rates arise because fitting with the standard RL model (Eqn. 4) approximates bipartite linear regression on the nonlinear value function. As a result, NRL-generated data will demonstrate a consistent relationship between apparent learning rate asymmetry and behavioral risk aversion (Fig. 3B). However, this relationship is driven by the strong relationship between risk preference and the generating NRL model $\sigma$ (Fig. 3C).

\section{A computational mechanism for distributional RL}

Asymmetric NRL prediction errors also provide a computational mechanism for recent theories of distributional reinforcement learning. In contrast to standard RL approaches, where models learn a single scalar quantity representing the mean reward, distributional RL models learn about the full distribution of possible rewards (Bellemare, Dabney, \& Munos, 2017; Dabney, Rowland, Bellemare, \& Munos, 2018). The critical difference in distributional approaches is a diversity of RPE channels, with differing degrees of optimism about outcomes, which learn varying predictions about future reward. While most theoretical work relies on learning rate differences to produce RPE asymmetries, a recent report shows that dopamine neurons themselves exhibit sufficient characteristics to support distributional RL (Dabney et al., 2020): (1) dopamine neurons show a diversity of reversal points (reward magnitude where prediction errors switch from negative to positive), (2) dopamine neurons differ in their relative weighting of positive and negative RPEs, (3) this asymmetry in RPE weighting correlates with reversal point across neurons, and (4) the diversity in reversal points and asymmetries support a decoding of reward distributions. Notably, these findings show that distributional learning arises from asymmetries in RPE coding rather than in downstream learning rates, but do not address how such asymmetry might arise.

Here, we show that - given parametric diversity in individual dopamine neurons - NRL provides a computational mechanism for the neurophysiological characteristics supporting distributional RL. In contrast to standard RL, distributional RL posits that different RPE channels learn different value predictions in the identical reward environment. In a variable outcome environment (Eshel et al., 2015; Eshel et al., 2016), this predicts that individual dopamine neurons will exhibit different reversal points. 
163

164

165

166

167

168

169

170

171

172

173

174

175

176

177

178

179

180

181

182

183

184

185

186

187

188

189

190

191

192

193

194

195

196

197

198

199

200

201

202

203

204

205

206

207

208

209

210

211

212

Recent work shows that empirical dopamine responses exhibit this hypothesized variability in reversal points, driven by asymmetric scaling of negative and positive RPE responses (Dabney et al., 2020). Given that such asymmetric RPE responses are intrinsic to NRL, we find that NRL agents simulated in such a stochastic reward environment exhibit a diversity of reversal points (examples, Fig. 4A) directly related to the individual NRL channel $\sigma$ parameter (Fig. 4B).

Moreover, the intrinsic RPE asymmetry of NRL model unit replicates key aspects of recorded dopamine neuron activity. First, NRL model units display a diversity of RPE biases, ranging from pessimistic (stronger negative RPE responses) to optimistic (stronger positive RPE responses) (Fig. 4C). Second, the degree of RPE asymmetry is directly related to average expected value learned in the simulated environment (as quantified by reversal points; Fig. 4D); this relationship mirrors that reported for recorded dopamine neurons. Finally, as a test of the ability of NRL to support distributional decoding, we examined whether a population of diverse NRL agents carries the necessary information to decode the distribution of previously experienced rewards (Fig. 4E). Specifically, as done in recent work on empirical dopaminergic responses, we assumed that NRL reversal points and response asymmetries learned in response to different reward environments - define a set of expectiles, and we transformed these expectiles into a probability density (Dabney et al., 2020). Following reward learning in a small population of NRL agents ( $n=40$, matched to empirical data), the probabilistic density of experienced rewards in different environments - including symmetric, asymmetric, or multimodal reward distributions - can be decoded from NRL responses (see Materials and Methods and Appendix). Thus, the NRL algorithm replicates diverse features observed in dopaminergic neurons, and provides a robust computational mechanism for distributional RL.

\section{Alternative parameterizations and biological plausibility}

While for simplicity we parameterize NRL heterogeneity above via the semisaturation term $\sigma$, equivalent variability in RPE asymmetry can be generated by differential weighting of reward inputs:

$$
V_{t+1}=V_{t}+\eta\left(\frac{\left(w R_{t}\right)^{n}}{\sigma^{n}+\left(w R_{t}\right)^{n}}-V_{t}\right)
$$

Because an increase in input weighting is equivalent to a decrease in effective $\sigma$ (Appendix), the $w$ term also controls RPE asymmetry: positive (negative) RPE biases occur for the same reward $R$ given sufficiently small (large) $w$ weights. This alternative formulation provides two additional benefits. First, it preserves the ability of the NRL model to capture adaptation, typically implemented in normalization approaches via a history-dependent $\sigma$ (e.g. average reward in the environment) (Heeger, 1992; Sinz \& Bethge, 2013). By separating how the NRL algorithm models RPE asymmetry and reward history, this parameterization can be used to examine neural and behavioral adaptation effects during reward learning. Second, variable input weighting is consistent with evidence for heterogeneity in both synaptic physiology and inputs to dopaminergic neurons (Morales \& Margolis, 2017). Differences in input weighting offers a more biologically plausible source of heterogeneity than the semisaturation term, which is typically considered a shared network property (i.e. baseline activity in a normalization pool) in circuit models of normalization (Carandini \& Heeger, 2012; Heeger, 1992).

\section{Discussion}

Standard RL algorithms assume linear reward representations, but the brain represents objective rewards in a subjectively nonlinear manner; here we show that a nonlinear RL algorithm - employing the canonical divisive normalization computation - captures diverse neural and behavioral features of reward learning and decision-making. While the use of nonlinear reward transformations in RL is not novel, normalization generates both convex and concave regimes and, as a result, asymmetries in RPE responses for negative and positive prediction errors. In addition to matching empirical observations of saturating, 
nonlinear reward representations, the NRL model explain aspects of observed behavioral and neural data including variable risk preferences and asymmetric RPE responses thought to support distributional RL.

Our results show that intrinsic asymmetries in reward learning can arise from subtleties in RPE coding rather than in downstream learning rates. However, these results do not preclude coexisting asymmetries in both systems. Both variability in dopaminergic genes and pharmacologic dopamine modulation affect reward learning in a valence-dependent manner, consistent with differential responses in striatal D1 and D2 receptors to positive versus negative RPEs (Frank et al., 2007; Frank et al., 2004). Such downstream differences would drive differential weighting of prediction errors carried by dopaminergic inputs. On the other hand, increasing evidence shows valence-dependent differences in dopamine neuron responses (Dabney et al., 2020; Fiorillo, Yun, \& Song, 2013), arguing for asymmetries in RPE coding itself. We suggest that NRL provides a computational mechanism to explain such asymmetries at the level of dopamine RPE representation, but valence-dependent biases in both prediction error coding and downstream processing likely play a role in biological learning.

While distributional RL has been proposed and implemented in computational algorithms, only recently has evidence arisen that dopamine neurons exhibit the necessary reward asymmetries (Dabney et al., 2020). Specifically, individual dopamine neurons differentially weight positive versus negative RPEs, and this relative valence-dependent weighting varies across neurons. However, how valence-dependent RPE coding arises is unknown. We show here that an RL system with a biologically-inspired normalized value function reproduces heterogeneous RPE asymmetries. In the NRL algorithm, asymmetry arises from the intrinsic curvature changes in the normalized reward function, and structured diversity in this asymmetry arises from parametric differences in normalized reward coding. Importantly, both asymmetry and structured diversity have plausible biological sources: normalization is produced by a number of mechanisms including feedforward inhibition, feedback inhibition, and synaptic depression (Carandini \& Heeger, 2012), and asymmetry diversity requires only heterogeneity in input weighting (e.g. synaptic strength). Thus, RPE asymmetry in NRL arises solely from the reward processing circuit, and does not require differential weighting of separate sources of negative and positive RPE information.

Variability in RPE asymmetries is crucial to theories of distributional learning based on expectile regression, and empirical dopamine RPE asymmetries carry sufficient information to decode distributional information about experienced rewards via expectile-based decoding methods. Our results show that a population of NRL agents can learn and encode sufficient information to allow decoding of experienced reward distributions, at a level comparable to decoding from empirical responses (Dabney et al., 2020). However, while this shows that distributional information exists in both empirical and NRL responses, it is unclear whether expectile-based decoding is biologically plausible or employed by the brain. Alternative distributional codes have been suggested to be more computationally straightforward (Tano, Dayan, \& Pouget, 2020); interestingly, these codes rely on variable nonlinear reward functions closely related to NRL responses, suggesting that NRL function in distributional learning may extend beyond expectile-based approaches. Further work is needed to verify distributional reward coding in downstream brain areas, identify the biological decoding algorithm, and test the contribution of NRL asymmetries to distributional learning.

Beyond capturing variability in risk preference and RPE asymmetry, the NRL model makes a number of further predictions about reward-guided behavior and neural activity. At the behavioral level, in addition to the across-subject variability shown here, the sigmoidal shape of the NRL value function predicts within-subject changes in risk preference. Under normalized value coding, the local curvature of the reward function depends on the relationship between rewards and the semisaturation term $\sigma$. Specifically, NRL predicts that individual risk preference should be magnitude dependent, with increasing risk aversion at larger outcomes; such outcome-dependent changes are consistent with some behavioral evidence (Fehr-Duda, Bruhin, Epper, \& Schubert, 2010; Holt \& Laury, 2002), but remains to be tested in 
strict reinforcement learning scenarios. At the neural level, NRL predicts an adaptive flexibility in individual (and population) dopamine neuron asymmetries. Unlike in other theories (Dabney et al., 2020), RPE bias in a given NRL agent depends on the reward magnitude (relative to $\sigma$ ). Thus, while a population of NRL agents should retain their relative ranking of asymmetries in different environments, absolute asymmetries will change depending on the experienced rewards - an effect that may confer an advantageous adaptability. More broadly, when RPE asymmetry diversity is parameterized by input weights (Eq. 4), the NRL algorithm can incorporate past reward information via a history-dependent $\sigma$ term. This suggests that NRL responses should capture contextual phenomena such as adaptive coding of reward values (Tobler, Fiorillo, \& Schultz, 2005) and adaptation in risk preferences (Rigoli, Rutledge, Dayan, \& Dolan, 2016).

In summary, we present a model of reinforcement learning that incorporates a biologically-relevant nonlinear reward function implemented by divisive normalization. Normalized value coding introduces a parametrically tunable valence-based bias in prediction errors, and structured diversity in this bias captures both variable risk preferences across individuals and variable prediction error weighting across neurons. Together, these findings reconcile empirical and theoretical aspects of reinforcement learning, support the robustness of normalization-based value coding, and argue for the incorporation of biologically valid value representations into computational models of reward learning and choice behavior.

\section{Materials and Methods}

\section{NRL model}

The NRL model applies a divisive normalization transform to experienced rewards (Eqn. 2), parameterized by an exponent $n$ and semisaturation term $\sigma$. Analytic analysis of NRL curvature is provided in the Supplementary Information. For all simulations (other than Fig. 1B), we fixed $n=2$. For simplicity, we implemented single state RL models that update value estimates with the product of the RPE and learning rate $\eta$ (Eqn. 3); however, the NRL framework can be applied in more complicated models that incorporate features like action policy updating and temporal difference learning(Sutton \& Barto, 1998). To examine RPE asymmetry, we quantified the response of different NRL agents (parameterized by varying $\sigma ; \eta=0.1$ ) to a fixed reward signal ( $R=50$ A.U.) corrupted with uniform noise (-40 to 40 A.U.). The degree of RPE asymmetry was quantified by piecewise linear regression analyses of negative and positive reward errors. Changing the parameters of the reward noise did not affect the qualitative finding of variable RPE asymmetries (Appendix). All simulations and analyses were performed in MATLAB (R2015b).

\section{Risk-dependent decision-making}

To examine risk preferences in the NRL model, we simulated agent behavior in a learning and choice task previously used to examine human subject behavior(Niv et al., 2012). NRL agents were generated with random $\sigma$ parameters in the range $[10,80]$. In this task, each NRL agent chose between a certain option ( $100 \%$ chance of 20 A.U. reward) and a risky option (50\% chance of 0 or 40 A.U. reward); initial values for both options were set to 0 . In a given trial, choice was determined via a softmax function of estimated option values; to achieve a comparable level of choice stochasticity across different agents, the softmax temperature was inversely scaled with the $\sigma$ parameter. When chosen, the value of an option was updated based on received outcome according to Eqn. $3(\eta=0.1)$. For each NRL agent, we simulated behavior for 1000 trials. To examine how NRL agent behavior appears if linear reward functions are assumed, we fit NRL-generated behavior with an RL model with valence-dependent learning rates ( $\eta^{+}$and $\eta^{-}$; Eqn. 4) and quantified the apparent learning rate asymmetry $\left(\eta^{-}-\eta^{+}\right) /\left(\eta^{-}+\eta^{+}\right)$.

\section{Distributional RL}


To examine whether information about experienced reward distributions were encoded in learned NRL responses, we quantified NRL agent behavior in different reward environments. Rewards were drawn from symmetric, left-skewed, and right-skewed distributions; in addition, we examined an environment with seven equiprobable rewards $(0.1,0.3,1.2,2.5,5,10$, or 20 A.U.), replicating the conditions under which empirical dopamine neurons exhibit RPE asymmetries $(15,16,35)$. To facilitate comparison to empirical decoding performance, we examined $40 \mathrm{NRL}$ agents a diversity of semisaturation parameters (0.5 to 48 A.U.). For each environment, analytical steady state NRL functions and reversal points were calculated for each NRL agent (Appendix), but similar results were obtained in when NRL agents learned via sampling. Following identification of the reversal point, we estimated the RPE response asymmetry of each agent via separate linear regressions for negative and RPE responses around the reversal point.

Given a reversal point $R_{n}^{*}$ and RPE asymmetry $\tau_{n}$ for each NRL agent denoted by index $n$ (see Appendix), distribution decoding was performed as previously described for empirical dopamine data (Dabney et al., 2020). Briefly, these data were interpreted as expectiles, where the $\tau_{n}$-th expectile had the value $R_{n}^{*}$. Decoding consisted of an imputation method to find a probability density that best matched the set of expectiles. As previously described, the density was parametrized as a set of 100 reward samples and optimization was performed to minimize a loss function between reversal points, asymmetries, and reward sample locations; see Appendix for details.

Data availability

Simulation data presented in this paper are available at https://doi.org/ 10.17605/OSF.IO/E6T5Z.

\section{Code availability}

MATLAB code used for simulation and analysis of the NRL model are available at https://doi.org/ 10.17605/OSF.IO/E6T5Z.

\section{Acknowledgements}

We thank W. Asaad, S. Bucher, P. Glimcher, A. Lee, M. Mehta, and B. Shen for comments on the manuscript. This work was supported by the National Institute of Mental Health of the National Institutes of Health under award number R01MH104251. The content is solely the responsibility of the author and does not necessarily represent the official views of the National Institutes of Health.

\section{Competing interests}

The author declares no competing interests. 


\section{References}

Bayer, H. M., \& Glimcher, P. W. (2005). Midbrain dopamine neurons encode a quantitative reward prediction error signal. Neuron, 47(1), 129-141. doi:S0896-6273(05)00467-8 [pii]

10.1016/j.neuron.2005.05.020

Bellemare, M. G., Dabney, W., \& Munos, R. (2017). A distributional perspective on reinforcement learning. Paper presented at the International Conference on Machine Learning.

Botvinick, M. M., Niv, Y., \& Barto, A. G. (2009). Hierarchically organized behavior and its neural foundations: a reinforcement learning perspective. Cognition, 113(3), 262-280. doi:10.1016/j.cognition.2008.08.011

Busse, L., Wade, A. R., \& Carandini, M. (2009). Representation of concurrent stimuli by population activity in visual cortex. Neuron, 64(6), 931-942. doi:10.1016/j.neuron.2009.11.004

Carandini, M., \& Heeger, D. J. (2012). Normalization as a canonical neural computation. Nat Rev Neurosci, 13(1), 51-62. doi:10.1038/nrn3136

Caze, R. D., \& van der Meer, M. A. (2013). Adaptive properties of differential learning rates for positive and negative outcomes. Biol Cybern, 107(6), 711-719. doi:10.1007/s00422-013-0571-5

Cohen, J. Y., Haesler, S., Vong, L., Lowell, B. B., \& Uchida, N. (2012). Neuron-type-specific signals for reward and punishment in the ventral tegmental area. Nature, 482(7383), 85-88. doi:10.1038/nature10754

Dabney, W., Kurth-Nelson, Z., Uchida, N., Starkweather, C. K., Hassabis, D., Munos, R., \& Botvinick, M. (2020). A distributional code for value in dopamine-based reinforcement learning. Nature, 577(7792), 671-675.

Dabney, W., Rowland, M., Bellemare, M. G., \& Munos, R. (2018). Distributional reinforcement learning with quantile regression. Paper presented at the AAAI Conference on Artifical Intelligence.

Daw, N. D., Kakade, S., \& Dayan, P. (2002). Opponent interactions between serotonin and dopamine. Neural Netw, 15(4-6), 603-616.

Dolan, R. J., \& Dayan, P. (2013). Goals and habits in the brain. Neuron, 80(2), 312-325. doi:10.1016/j.neuron.2013.09.007

Eshel, N., Bukwich, M., Rao, V., Hemmelder, V., Tian, J., \& Uchida, N. (2015). Arithmetic and local circuitry underlying dopamine prediction errors. Nature, 525(7568), 243-246. doi:10.1038/nature14855

Eshel, N., Tian, J., Bukwich, M., \& Uchida, N. (2016). Dopamine neurons share common response function for reward prediction error. Nat Neurosci, 19(3), 479-486. doi:10.1038/nn.4239

Fehr-Duda, H., Bruhin, A., Epper, T., \& Schubert, R. (2010). Rationality on the rise: Why relative risk aversion increases with stake size. Journal of Risk and Uncertainty, 40(2), 147-180.

Fiorillo, C. D., Yun, S. R., \& Song, M. R. (2013). Diversity and homogeneity in responses of midbrain dopamine neurons. Journal of Neuroscience, 33(11), 4693-4709. 
Frank, M. J., Moustafa, A. A., Haughey, H. M., Curran, T., \& Hutchison, K. E. (2007). Genetic triple dissociation reveals multiple roles for dopamine in reinforcement learning. Proc Natl Acad Sci U $S$ A, 104(41), 16311-16316. doi:10.1073/pnas.0706111104

Frank, M. J., Seeberger, L. C., \& O'Reilly R, C. (2004). By carrot or by stick: cognitive reinforcement learning in parkinsonism. Science, 306(5703), 1940-1943. doi:10.1126/science.1102941

Gershman, S. J. (2015). Do learning rates adapt to the distribution of rewards? Psychon Bull Rev, 22(5), 1320-1327. doi:10.3758/s13423-014-0790-3

Heeger, D. J. (1992). Normalization of cell responses in cat striate cortex. Vis Neurosci, 9(2), 181-197. doi:10.1017/S0952523800009640

Holt, C. A., \& Laury, S. K. (2002). Risk aversion and incentive effects. American Economic Review, 92(5), 1644-1655.

Lefebvre, G., Lebreton, M., Meyniel, F., Bourgeois-Gironde, S., \& Palminteri, S. (2017). Behavioural and neural characterization of optimistic reinforcement learning. Nature Human Behaviour, 1(4), 0067.

Levy, I., Snell, J., Nelson, A. J., Rustichini, A., \& Glimcher, P. W. (2010). Neural representation of subjective value under risk and ambiguity. $J$ Neurophysiol, 103(2), 1036-1047. doi:10.1152/jn.00853.2009

Louie, K., Grattan, L. E., \& Glimcher, P. W. (2011). Reward value-based gain control: divisive normalization in parietal cortex. J Neurosci, 31(29), 10627-10639. doi:31/29/10627 [pii]

10.1523/JNEUROSCI.1237-11.2011

Luce, R. D. (1959). Individual Choice Behavior: A Theoretical Analysis. New York: Wiley.

Mihatsch, O., \& Neuneier, R. (2002). Risk-sensitive reinforcement learning. Machine Learning, 49(2-3), 267-290.

Mnih, V., Kavukcuoglu, K., Silver, D., Rusu, A. A., Veness, J., Bellemare, M. G., . . Hassabis, D. (2015). Human-level control through deep reinforcement learning. Nature, 518(7540), 529-533. doi:10.1038/nature14236

Morales, M., \& Margolis, E. B. (2017). Ventral tegmental area: cellular heterogeneity, connectivity and behaviour. Nature Reviews Neuroscience, 18(2), 73.

Niv, Y., Edlund, J. A., Dayan, P., \& O'Doherty, J. P. (2012). Neural prediction errors reveal a risksensitive reinforcement-learning process in the human brain. $J$ Neurosci, 32(2), 551-562. doi:10.1523/JNEUROSCI.5498-10.2012

Ohshiro, T., Angelaki, D. E., \& DeAngelis, G. C. (2017). A Neural Signature of Divisive Normalization at the Level of Multisensory Integration in Primate Cortex. Neuron, 95(2), 399-411 e398. doi:10.1016/j.neuron.2017.06.043

Padoa-Schioppa, C., \& Assad, J. A. (2006). Neurons in the orbitofrontal cortex encode economic value. Nature, 441(7090), 223-226. doi:nature04676 [pii]

10.1038/nature04676 
Palminteri, S., Lefebvre, G., Kilford, E. J., \& Blakemore, S. J. (2017). Confirmation bias in human reinforcement learning: Evidence from counterfactual feedback processing. PLoS Comput Biol, 13(8), e1005684. doi:10.1371/journal.pcbi.1005684

Reynolds, J. H., \& Heeger, D. J. (2009). The normalization model of attention. Neuron, 61(2), 168-185. doi:S0896-6273(09)00003-8 [pii]

10.1016/j.neuron.2009.01.002

Rigoli, F., Rutledge, R. B., Dayan, P., \& Dolan, R. J. (2016). The influence of contextual reward statistics on risk preference. Neuroimage, 128, 74-84. doi:10.1016/j.neuroimage.2015.12.016

Schultz, W., Dayan, P., \& Montague, P. R. (1997). A neural substrate of prediction and reward. Science, 275(5306), 1593-1599.

Sheahan, H., Luyckx, F., Nelli, S., Teupe, C., \& Summerfield, C. (2021). Neural state space alignment for magnitude generalization in humans and recurrent networks. Neuron.

Silver, D., Schrittwieser, J., Simonyan, K., Antonoglou, I., Huang, A., Guez, A., . . Hassabis, D. (2017). Mastering the game of Go without human knowledge. Nature, 550(7676), 354-359. doi:10.1038/nature24270

Sinz, F., \& Bethge, M. (2013). Temporal adaptation enhances efficient contrast gain control on natural images. PLoS Comput Biol, 9(1), e1002889. doi:10.1371/journal.pcbi.1002889

Song, H. F., Yang, G. R., \& Wang, X. J. (2017). Reward-based training of recurrent neural networks for cognitive and value-based tasks. Elife, 6. doi:10.7554/eLife.21492

Stauffer, W. R., Lak, A., \& Schultz, W. (2014). Dopamine reward prediction error responses reflect marginal utility. Curr Biol, 24(21), 2491-2500. doi:10.1016/j.cub.2014.08.064

Sutton, R. S., \& Barto, A. G. (1998). Reinforcement Learning : An Introduction. Cambridge, MA: MIT Press.

Tano, P., Dayan, P., \& Pouget, A. (2020). A local temporal difference code for distributional reinforcement learning. Paper presented at the Advances in Neural Information PRcoessing Systems 33.

Tobler, P. N., Fiorillo, C. D., \& Schultz, W. (2005). Adaptive coding of reward value by dopamine neurons. Science, 307(5715), 1642-1645. doi:307/5715/1642 [pii]

10.1126/science. 1105370

Von Neumann, J., \& Morgenstern, O. (1944). Theory of Games and Economic Behavior. Princeton, NJ: Princeton University Press. 
a

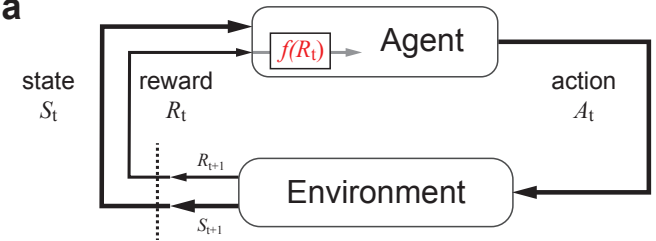

b
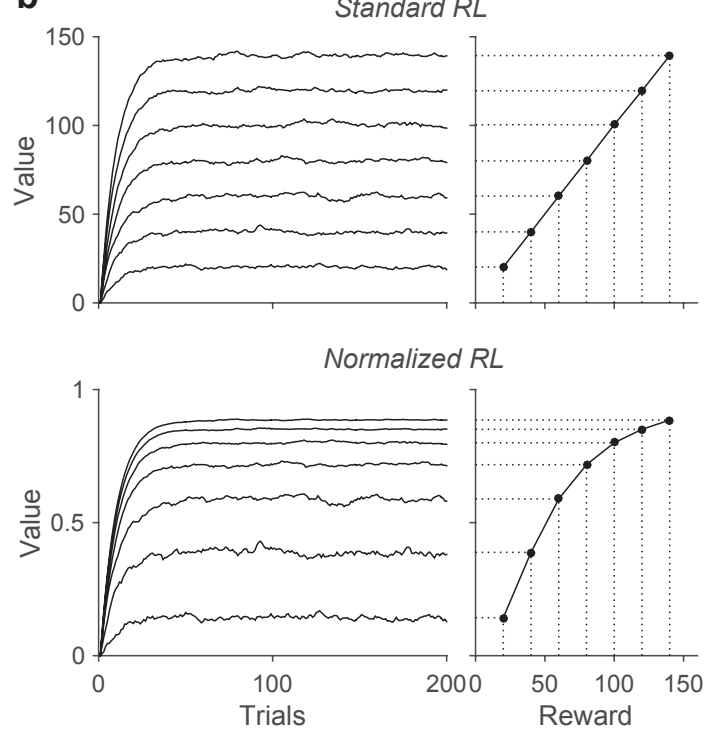

Standard RL

$V_{t+1}=V_{t}+\alpha\left(R_{t}-V_{t}\right)$

Normalized RL $\quad V_{t+1}=V_{t}+\alpha\left(\frac{R_{t}^{n}}{\sigma^{n}+R_{t}^{n}}-V_{t}\right)$

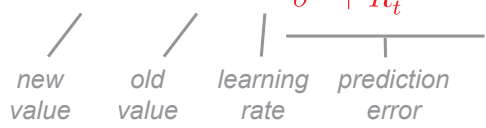

c

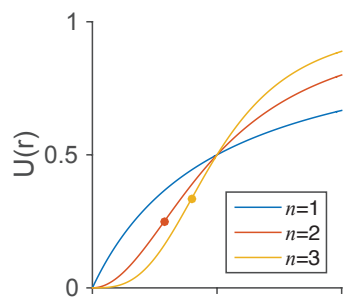

d
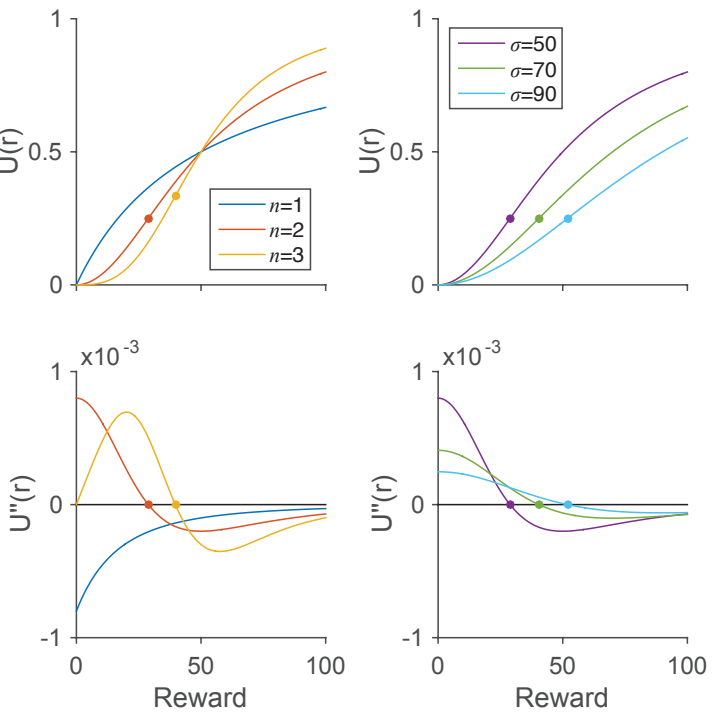

Figure 1. Normalized reinforcement learning model.

(a) Comparison of standard reinforcement learning (RL) and normalized reinforcement learning (NRL) models. RL and NRL differ in how external rewards are transformed by the reward coding function $f\left(R_{\mathrm{t}}\right)$ prior to learning internal value estimates. Standard RL uses a linear reward function, while NRL uses a divisively normalized representation. (b) Learned value functions under RL and NRL. Left, dynamic value estimates during learning. Right, steady state value estimates. Simulations were performed for each of seven rewards, with additive zero-mean Gaussian noise (learning rate $\alpha=0.1$ ). In contrast to RL, NRL algorithms learn values that are a nonlinear function of external rewards. (c) Convexity and concavity in NRL value functions. Top, NRL value functions with different exponents (fixed $\sigma=50$ A.U.). Bottom, second derivative of value functions. Dots show inflection points between convex and concave value regimes. (d) Parametric control of NRL value curvature. NRL value function (top) and second derivative (bottom) for different $\sigma$ values (fixed $n=2$ ). 

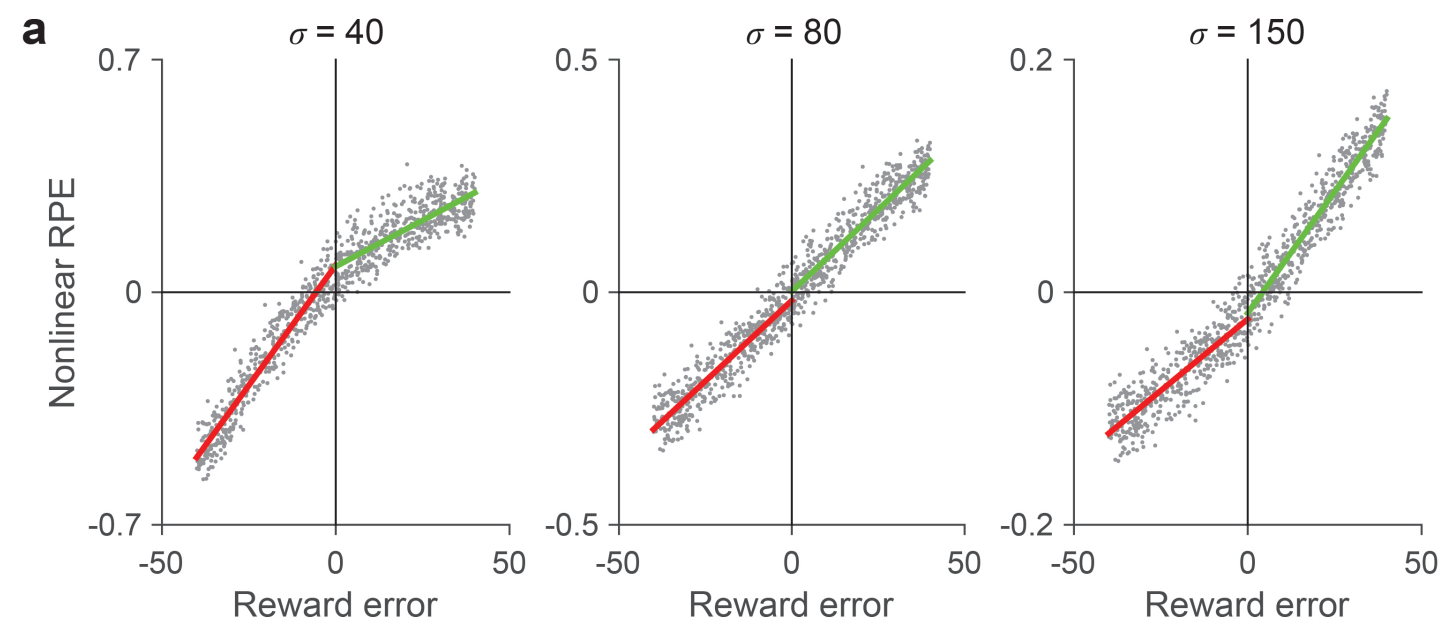

b

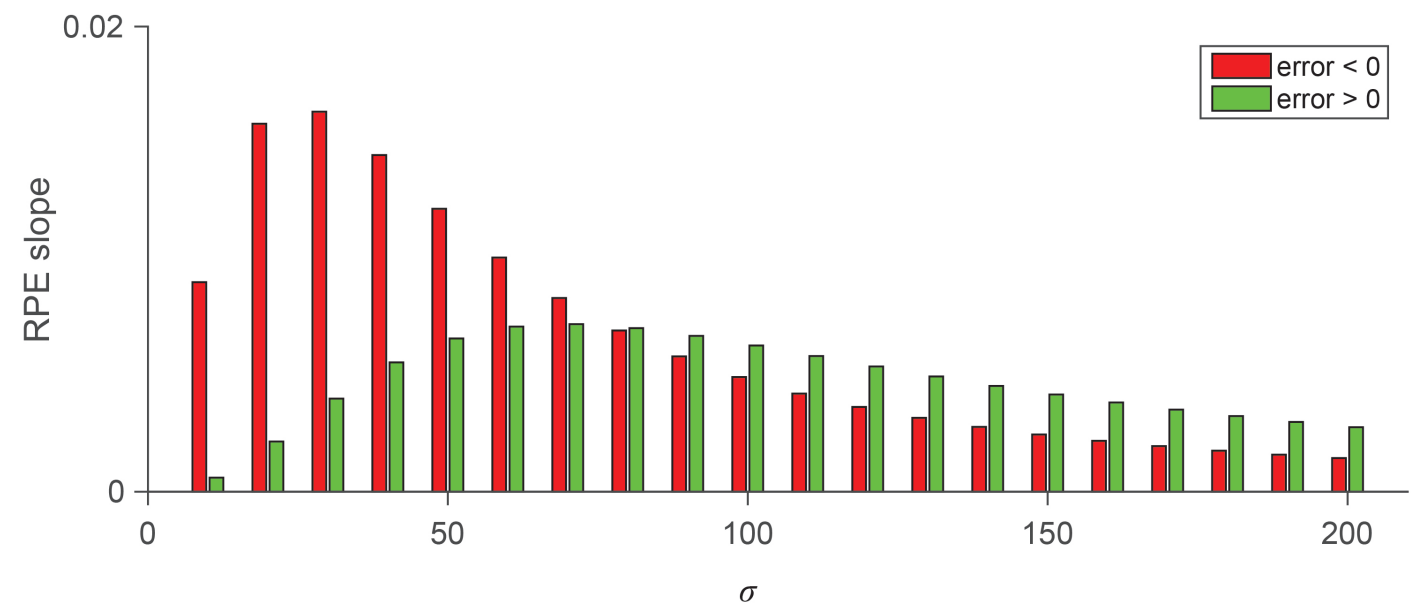

471

472

473

474

475

476

477
Figure 2. Parametric control of prediction error asymmetry.

(a) Examples of variable reward prediction error (RPE) asymmetry. Each panel shows NRL responses for reward inputs $(R=50$ A.U.) with uniformly distributed noise. Lines show piecewise linear regression fits for negative (red) and positive (green) reward errors. (b) The NRL semisaturation term governs the degree and direction of RPE asymmetry. NRL RPE asymmetry is biased towards negative RPEs at low $\sigma$ and postive RPEs at high $\sigma$. 

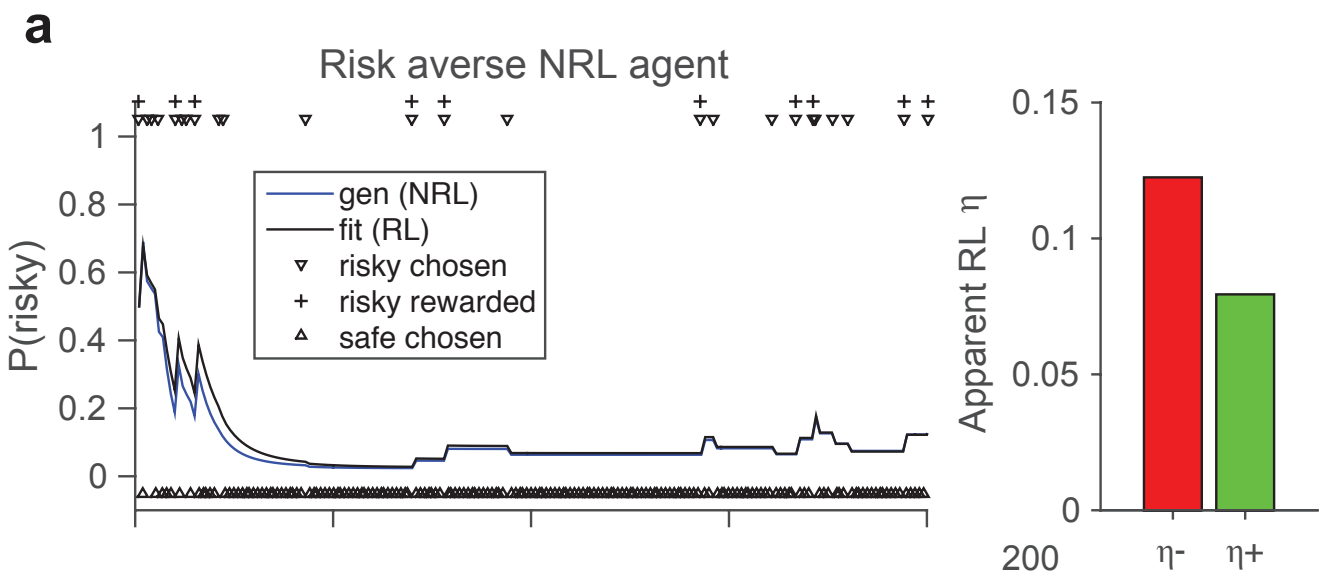

Risk seeking NRL agent
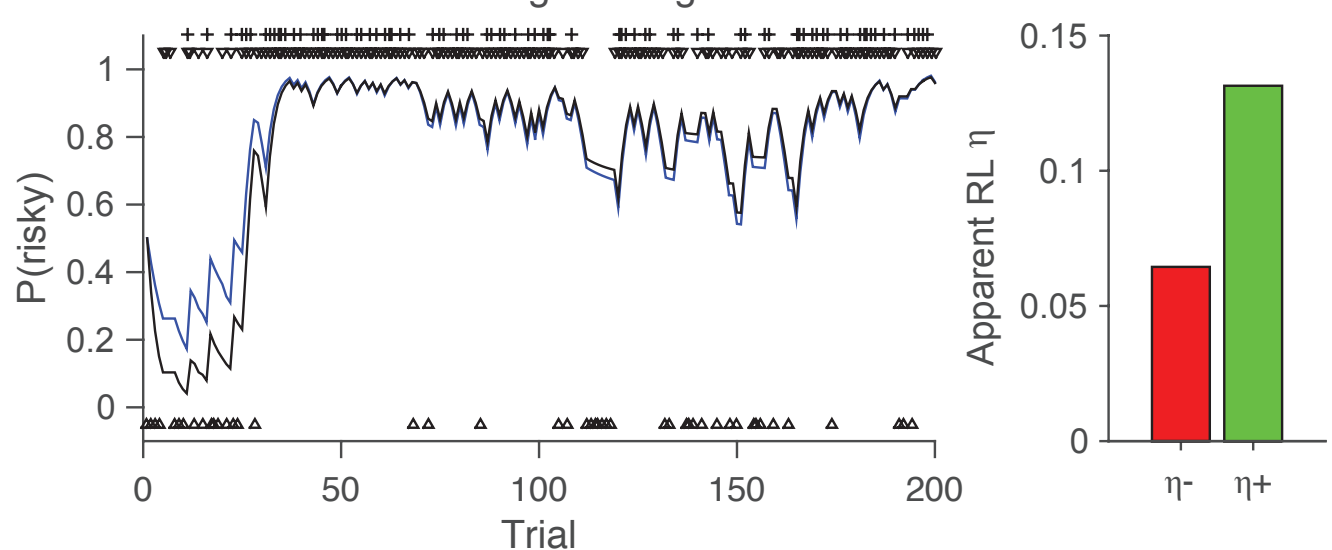

b

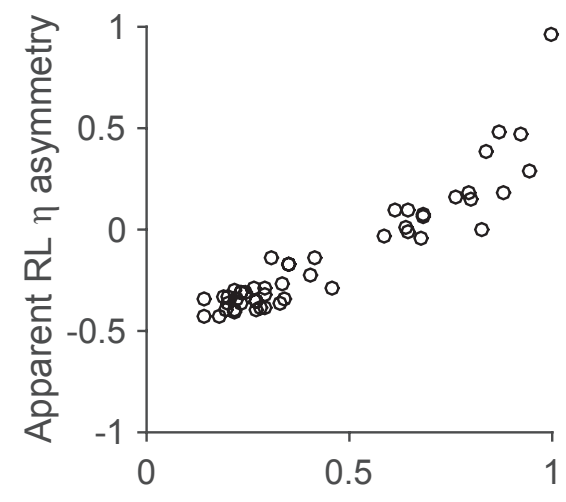

Behavioral risk aversion

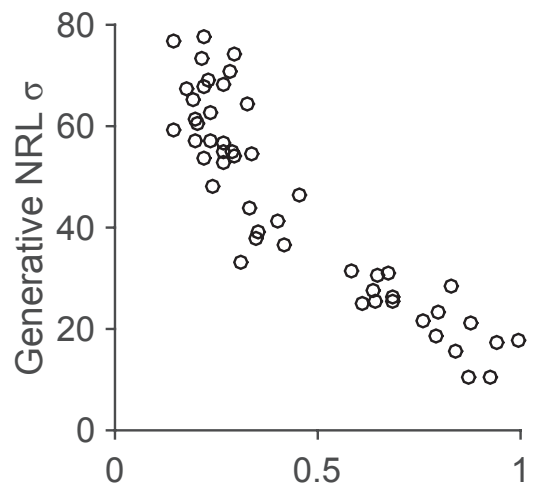

Behavioral risk aversion
Figure 3. NRL RPE asymmetry governs the degree of risk preference in reward learning.

(a) Examples of risk averse and risk seeking NRL agent behavior. Left, behavior in a task involving choices between a certain ( $100 \%$ chance of 20 A.U.) and a risky (50\% chance of 0 or 40 A.U.) option. Blue lines, behavior of the generative NRL agent. Black lines, behavior of best fitting linear RL model with asymmetric learning rates. Right, apparent learning rates for negative and positive RPEs in linear RL model. (b) Apparent relationship between risk preference and asymmetric learning rates under 
assumption of linear reward coding. Behavioral risk aversion (percent choice of certain option) and NRL semisaturation parameter. 
b
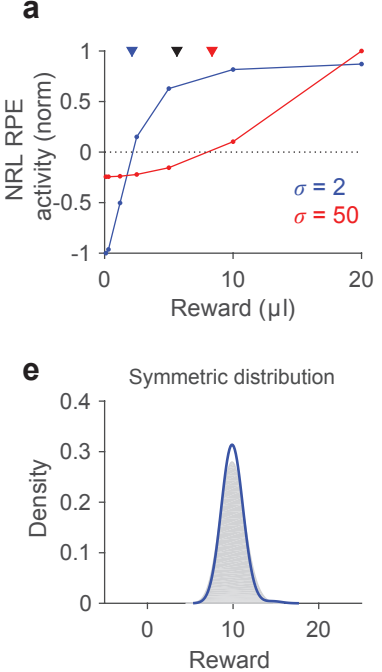
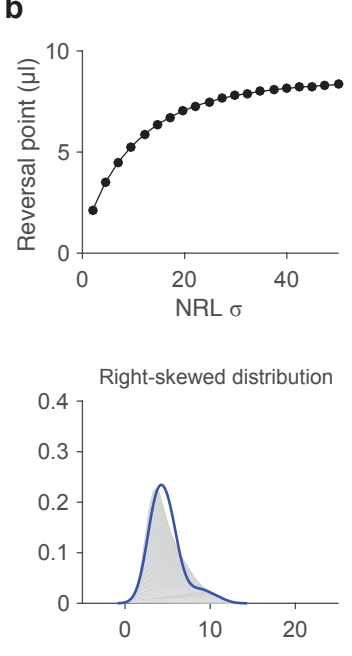
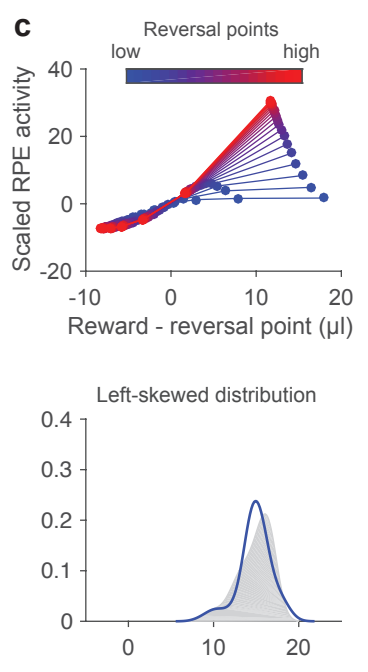
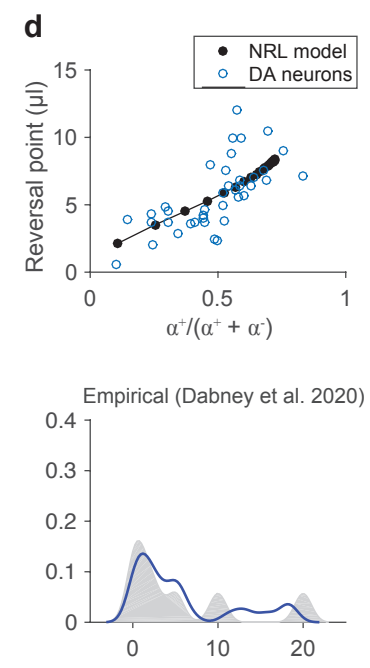

Figure 4. NRL RPE asymmetry provides a computational basis for distributional reinforcement learning.

(a) Variable NRL RPE response asymmetries in a probabilistic reward environment. Examples show NRL agents with stronger negative (blue) and positive (red) RPE asymmetry. Note that these two agents exhibit different reversal points in the same reward environment (rewards $=\{0.1,0.3,1.2,2.5,5,10,20$ $\mu l\}$, as in previous work (Dabney et al., 2020)). Triangles denote the true average reward (black) and estimated average reward learned by pessimistic (blue) and optimistic (red) NRL agents. (b) Learned reversal points vary systematically with NRL parameterization. RPE responses and reversal points quantified for varying $\sigma$ parameters. (c) Reversal points depend on NRL RPE asymmetry. Plots show NRL responses normalized by negative RPE slope and aligned to individual reversal points. As in empirical dopamine data, low (high) reversal points arise from stronger negative (positive) RPE asymmetry. (d) NRL asymmetry and learning match empirical dopamine data. Blue, dopamine neurons recorded in stochastic reward environment (Dabney et al., 2020); black, heterogeneous NRL agents in identical reward environment. Asymmetry is defined as in previous work as a function of positive $\left(\alpha^{+}\right)$ and negative $\left(\alpha^{-}\right)$RPE coding slopes. (e) A population of NRL agents learns the distribution of experienced rewards. $40 \mathrm{NRL}$ agents were simulated in four different reward environments: symmetric, right-skewed, left-skewed, and multimodal. Each panel plots the ground truth (gray) and decoded (blue) probability densities, with samples smoothed by kernel density estimation. Distribution decoding was performed via an imputation strategy, treating the NRL reversal points and response asymmetries as expectiles. 